\title{
Representaciones que subyacen a la política educacional inclusiva en Chile
}

\author{
Representations that underlay the inclusive educational policy in Chile
}

\author{
Jorge Eduardo Alfaro-Urrutia / jorgeeduardoau@gmail.com \\ orcid.org/0000-0003-3711-6536 \\ Universidad de la Frontera, Chile
}

\begin{abstract}
The implementation of Chilean inclusive education policy has not been without discussion. Structuring a differenced and open education system faced the stresses of uncertainty. In this context, based on a qualitative methodology, this study discusses the social representations of inclusive policy from 378 online comments posted by Internet users of Chilean news portals. Content analysis was performed to identify the assessments and arguments supporting the representations of educational inclusion. The inability to counterargument in past virtual environments is identified as the main limitation for in-depth information. Two models of representations are identified; the first linked to a social reproduction orientation associated to learning disciplinary standards and assessment of institutional educational projects, while the second assessed the right to education prioritizing learning and respect for individuals.
\end{abstract}

Key words: educational policy, representations, inclusive education, social transformation, discrimination.

Resumen: La implementación de la política educacional inclusiva chilena no ha estado exenta de discusión. La intención de estructurar un sistema educativo con apertura a las diferencias, tensiona la incertidumbre con la necesidad de cambio. En este contexto, se pretende discutir representaciones respecto de la política inclusiva chilena, para lo cual, desde una metodología cualitativa y a través de un análisis de contenido, se revisaron 378 comentarios online publicados por usuarios de portales noticiosos. Se identificaron las valoraciones y argumentos que sustentan las representaciones respecto de las diferencias en el contexto educativo. La imposibilidad de contraargumentar en entornos virtuales se identifica como la principal limitación para profundizar información. Se identifican dos modelos de representaciones: uno vinculado con un sistema educativo orientado hacia la reproducción social que asocia el aprendizaje a normas disciplinares y a la valoración de los proyectos educativos institucionales; y otro que valora el derecho a la educación, priorizando el aprendizaje y el respeto a las individualidades.

Palabras clave: política educativa, representaciones, inclusión educativa, transformación social, discriminación. 


\section{Introducción $^{1}$}

Durante el siglo XX, diversas convenciones internacionales situaron el problema de la desigualdad educativa (ONU, 1948; Unicef, 1989; Unesco, 1990). En este contexto se promovieron iniciativas orientadas a satisfacer las necesidades de aprendizaje, universalizar el acceso a la educación, concentrar la atención en el aprendizaje, ampliar los alcances de la educación y fortalecer todas las acciones orientadas a ella. Sin embargo, las convenciones del siglo XXI no han logrado demostrar progresos universales en la materia (Unesco, 2015 y 2005), situando a América Latina como una de las regiones más desiguales del planeta (OECD, 2016). La necesidad de analizar la situación de cada país en esta materia se torna relevante para promover los cambios requeridos.

El contexto chileno explica este fenómeno de desigualdad socioeducativa en la Constitución y normativa promovida en periodo de dictadura, la cual armonizó el rol del Estado con el del mercado, y que en los años siguientes de democracia, siguió concretando sus esfuerzos sobre dicha base (Oliva, 2010; Cox, 2012, Donoso-Díaz et al., 2012). En este sentido, la política educativa chilena se ha sustentado sobre la racionalidad económica, en la cual el aprendizaje se entiende como producto de canje en el mercado (Bourdieu, 1997; Cavieres, 2014). Así, las políticas de financiamiento y de gestión centralizada se identifican como factor que contribuye a la segmentación social (Castillo et al., 2011; Donoso-Díaz y Arias, 2012), transformándose en un desafío articular un sistema equitativo sobre un sistema de mercado.

A causa de lo anterior, se ha situado a Chile como el país de la Organización para la Cooperación y el Desarrollo Económico con mayor desigualdad de ingresos según el Coeficiente de Gini (OECD, 2015). Por su parte, Bellei (2013) ha establecido que la segregación académica y socioeconómica chilena es significativamente alta entre los estudiantes, lo cual es causa y consecuencia de la desigualdad nacional. Las medidas de selección educativa se han naturalizado, haciendo posible la selección, diferenciación, clasificación y jerarquización de estudiantes y maestros (Vásquez, 2015; Villalobos y Quaresma, 2015).

Se promueve así una cultura donde "se ha internalizado la idea de que las escuelas no deben hacer nada por mejorar la integración y que tampoco se puede imponer por ley" (Treviño et al., 2011:44). Así descrito, el rol de

1 El presente trabajo fue financiado con recursos de la Comisión Nacional de Investigación Científica y Tecnológica CONICYT del Gobierno de Chile, a través de su Beca de Doctorado Nacional. 
la educación chilena promueve disparidades y límites a la movilidad social (Araujo y Martucelli, 2015), en el cual parece entenderse que el ser diferente influirá negativamente en los resultados (Ortiz, 2014).

La transición del Estado chileno, desde un modelo de mercado educacional (años ochenta), pasando por un modelo híbrido entre mercado y política educativa (años noventa), lo ha llevado a situarse hoy desde un enfoque de derecho (Bellei, 2015). Sin embargo, la falta de prácticas reflexivas se ha identificado como un elemento que, en conjunto con las lógicas objetivas de los sistemas, obstaculiza los cambios culturales (Salvat, 2009).

En este contexto, el análisis de instrumentos legales chilenos vinculados al proceso de inclusión evidencia que las normativas promueven diagnósticos, clasificaciones y compensaciones que restringen a los sujetos como portadores de una diferencia, que se reduce para su asimilación (Infante, 2010; Peña, 2013). Asimismo, Moraga (2015) ha identificado respecto de la recientemente promulgada Ley 20845 de Inclusión Escolar en marzo de 2016, que los cambios normativos chilenos no modifican la estructura económica y competitiva, cumpliendo un rol de instruir y orientar más que transformar la cultura.

$\mathrm{Al}$ respecto, se ha establecido que crear culturas inclusivas implica trabajar creencias y supuestos personales en tanto las instituciones presentan barreras internas (rigidez curricular, sistemas de evaluación, capacitación de profesores, representaciones sociales) y barreras externas (procesos culturales discriminatorios y niveles socioeconómicos) (Blanco, 2014). De lo anterior, se desprende que la inclusión educativa trasciende hacia aspectos valóricos de la sociedad, discurso, reflexión y evaluación crítica (McCaster, 2015), a raíz de lo cual se logra una visión filosófica institucional para superar la exclusión y promover la participación, aprendizaje y sentimiento de pertenencia de los miembros de la comunidad (Booth, 1995; Mittler, 1995; Ainscow, 2005; Salend, 2011).

Las barreras que obstaculizan los procesos inclusivos han sido descritas desde las formas de poder que subordinan a aquel que es diferente y a sus formas de conocer (Santos, 2005 y 2010). En este sentido, los patrones de poder hegemónicos han situado formas de colonialismo, modernidad y eurocentrismo que polarizan la situación social y mercantiliza tanto la subjetividad como la vida de los sujetos. Se promueve así la clasificación e identificación racial, sexual y/o social de la población (Quijano, 2014). Lo anterior demanda una visión crítica del fenómeno educativo en vías de identificar las barreras que obstaculizan la inclusión y las formas de poder que subyacen a dichas barreras. 
En este sentido, Slee (2012) plantea que muchos esfuerzos por incluir no contribuyen al problema de la exclusión, sino que incluso aumentan las brechas. El modelo vinculado con la igualdad de oportunidades en la escuela "tiene como efecto acentuar la competencia escolar entre las familias a las que les interesa ahondar la brecha con el fin de asegurar las mejores oportunidades para sus hijos" (Dubet, 2014: 84). El mérito personal constituye un elemento a favor de la movilidad social; una vez que los sistemas reducen las desigualdades iniciales, es responsabilidad de los sujetos aprovechar las oportunidades para mejorar, lo cual implica necesariamente el fracaso de otros.

$\mathrm{Al}$ respecto, "el problema social y cultural es el individualismo competitivo" (Slee, 2011:80), el cual radica en un aspecto económico vinculado con las recesiones que exacerban el odio a las minorías. Pareciera entenderse, de acuerdo con estos planteamientos, que el otro diferente, quien no contribuye al crecimiento económico que nosotros queremos, se transforma en un problema social. En este contexto, Marguilis (1999) ha descrito que la identidad social opera por diferencia, en tanto la percepción del otro implica una comunicación que encuentra obstáculos para concretarse.

Esta mirada educativa, que tiene en su base a Durkheim, supone una escuela que reproduce patrones sociales y prepara a los individuos para tal sistema. Así lo plantea el autor cuando señala que "la educación perpetúa y refuerza esta homogeneidad fijando de antemano en el alma del niño las semejanzas esenciales que supone la vida colectiva" (Durkheim, 2002: 31). Como forma de responder a esta educación, el rol de la pedagogía se torna relevante para generar cambios.

Mehan (2008) sugiere dos visiones de la escolaridad, una meritocrática que idealiza a la escuela como institución que entrega oportunidades de acuerdo con los esfuerzos individuales, y otra donde las escuelas reproducen la estratificación existente en la sociedad. Por otra parte, se ha planteado una educación basada en la justicia que analice el currículum en busca de formas que atiendan las necesidades de todos los colectivos sociales (Sacristán, 2010; Torres, 2010), toda vez que la educación de masas abrió nuevas demandas de respuesta a la diversidad.

Estas ideologías sobre el currículum son entendidas, según Giroux (1983), como representaciones inscritas en la conducta, conciencia, discurso y experiencias, materializadas, a su vez, en el currículum educativo en forma de ideas respecto de la naturaleza de la educación, el saber, el aprendizaje y la enseñanza (Elliot, 2005).

En este sentido, Schiro (2008) plantea una ideología orientada hacia la reconstrucción social, la cual plantea como finalidad de la educación el 
favorecer una sociedad más justa y que ofrezca satisfacción a sus integrantes. De esta forma, los componentes ideológicos sobre los fines y medios de la educación cobran relevancia para comprender sus prácticas.

Se plantea, por tanto, la necesidad de enfrentar a los actores sociales con la finalidad de transparentar objetivos, puntos de vista y procedimientos basados en el diálogo y el consenso (Jobert, 2004). De esta forma, se pretende promover cambios, aunque éstos sean menores, pero con base en un amplio consenso, y no lo opuesto (Aguilar, 2000). Sin embargo, los efectos del mundo globalizado suponen una política sensible a la velocidad con que cambian las necesidades y demandas de la población (Tarabini-Castellani y Sarró, 2011). Así, los cambios sociales pueden no condecirse con cambios en materia de referenciales políticos, los cuales constituyen representaciones cognitivas, normativas e instrumentales sobre la realidad que subyacen a las políticas públicas (Jobert, 2004).

Lo anterior es coherente con el concepto de habitus, entendido como un "sistemas de disposiciones duraderas y transferibles, estructuras estructuradas predispuestas a funcionar como estructuras estructurantes" (Bourdieu, 1980: 86). Así, los habitus se estructuran a lo largo de las experiencias del sujeto inmerso en la sociedad y su cultura, y van sedimentando formas de entender los fenómenos a tal punto que se convierten en principios que organizan las prácticas. Por tal motivo, las prácticas no pueden explicarse "sino a condición de vincular las condiciones sociales en las que se ha constituido el habitus que las ha engendrado con las condiciones en que éste opera" (Bourdieu, 1980: 91).

De esta forma, se ha identificado que la educación chilena, como escenario simbólico donde el mercado y la masificación operan, se somete a procesos de diferenciación y desplazamiento (Corvalán, 2013). Lo anterior tensiona la política educativa entre la autonomía y el control social de individuos e instituciones, a través de una reflexión respecto de cómo las intervenciones favorecen el pensamiento crítico, la ampliación del universo sociocultural y la realización de los sujetos (Capriati, 2015).

De lo anteriormente expuesto y considerando la situación de la educación chilena, los desafíos para resolver la desigualdad y el difícil rol de la política educativa, para romper con los efectos de una historia de educación de mercado y de estructura social, se plantea como objetivo del presente trabajo describir las representaciones respecto de los cambios en materia de educación inclusiva, propuestos por la política chilena. Realizar este estudio permitirá desvelar los nudos críticos de la implementación política que tensionan valores, relaciones y sistemas cognitivos para desarrollar una educación más inclusiva, democrática y justa. 


\section{Método}

El presente artículo se desarrolla bajo un paradigma naturalista interpretativo (Crotty, 1998), un enfoque cualitativo (Gurdián-Fernández, 2007) y un diseño de etnografía virtual (Hine, 2004). En este sentido, el interés es describir un concepto social a través del análisis de las estructuras lingüísticas utilizadas para ello en entornos virtuales.

Para tales efectos se recopilaron comentarios de usuarios de cinco entornos virtuales conformados por artículos noticiosos respecto de la Ley de Inclusión Escolar. En este contexto, los usuarios publican comentarios sobre su apreciación con la mencionada ley. El entorno virtual como campo de investigación surge como un espacio de reformulación del objeto de estudio, centrado en el flujo y las conexiones más que en las localidades y los límites, como principios que organizan la investigación (Hine, 2004). Así, la investigación encuentra un nicho y nuevas fuentes para poder desarrollar su acción.

La necesidad de ampliar el rol del investigador en el ciberespacio, considerando que "las tecnologías de la información y la comunicación han generado muchas oportunidades para que las personas se pongan en contacto, interactúen, opinen y construyan en grupo" (Orellana y Sánchez, 2006: 76) se ha hecho manifiesta. Los vínculos que se dan en la cultura de Internet son coherentes con aquellos que se manifiestan en la sociedad, permitiendo utilizarlas como fuente de análisis para la investigación social (Sade-Beck, 2004; Welschinger, 2012) Con todo, aún se evidencian desafíos; Martínez (2013) plantea que en dichas plataformas las discusiones no se desarrollan de manera continua.

Considerando la posibilidad de utilizar redes sociales como oportunidad para desarrollar una investigación cualitativa, se procedió a la búsqueda de archivos noticioso que presentaran información respecto de la Ley de Inclusión Escolar durante el mes de febrero de 2016. Para tales efectos se seleccionaron cinco artículos de cinco medios de comunicación digital diferentes, que abordaron la temática y que presentaban discusión entre usuarios del medio. De estos artículos, se tomaron 378 comentarios realizados por los usuarios (véase Tabla $1^{2}$ ). Del total, 105 comentarios fueron hechos por personas de género femenino, 265 por personas de género masculino y 8 comentarios cuyo género no se pudo determinar a través de indicadores convencionales.

El procedimiento se estructuró en forma de grupo de discusión de Internet, entendido éste como un discurso que, a diferencia del grupo conven-

2 Todas las tablas se encuentran en el Anexo, al final del presente artículo (Nota el editor). 
cional, "se constituye en la medida que un conjunto de integrantes de una lista discuten un tema específico[...] y al cual se integran, durante un tiempo, algunos miembros que -interesados en el tema- responden a la provocación inicial" (Henríquez, 2002:78). Un estudio realizado por Callén et al. (2007) utilizó un método similar considerando comentarios en una lista de correo.

La situación ético confidencial, vinculado al consentimiento de los participantes, constituye un problema en tanto se asume que los comentarios son declaraciones públicas, lo cual no está exento de conflicto (Hine, 2004). Al considerar la imposibilidad de gestionar consentimientos individuales por lo insustentable de la comunidad, una solución consiste en "cambiar los nombres de usuarios, así como algunos rasgos que los identifiquen” (Hine, 2004: 37). Para efectos de esta investigación, si bien se conservaron las citas textuales, se omitió en su presentación cualquier nombre tanto del medio en que se reprodujo como del sujeto, así como aspectos vinculados a la identidad de la persona.

Los mensajes fueron transcritos de forma textual, conservando sintaxis y ortografía original. Posteriormente se procedió a analizarlos en el Software Atlas.Ti para Windows, a través de, en primera instancia, una codificación abierta que tuvo como finalidad determinar los conceptos centrales, sus propiedades y dimensiones respecto de referencias de los usuarios en torno a la política inclusiva. Después se realizó una codificación axial, en la cual se agruparon los conceptos emergentes estableciéndose las relaciones que permitieron dar respuesta al objetivo del artículo.

\section{Resultados}

De acuerdo con el análisis de los comentarios en cuanto a la política de inclusión chilena, se puede señalar que existe una polarización respecto de las opiniones, las cuales se concentran en aprobar o desaprobar la normativa. Si bien la Ley de Inclusión presenta distintas aristas, como el fin del copago y del lucro, los comentarios, se centran en torno a aspectos de responsabilidad e imagen de los estudiantes. La Tabla 2 presenta los elementos que conforman las representaciones sobre la política inclusiva chilena.

Respecto de los participantes que tienden a aprobar la ley de inclusión escolar, se puede señalar que muestran representaciones sobre la política inclusiva centradas en el aprendizaje del estudiante. En este sentido, se tiende a asumir que la imagen y búsqueda de identidad no influye en lo que aprende y, por tanto, no debieran ser reguladas por las instituciones educativas. 
Véalo desde el punto de vista, que ahora tendrá que centrarse sólo en transmitir el conocimiento, o bien generarlo en los estudiantes, y no tendrá que andar controlando cómo visten (Comentario 38, Medio 1).

Acaso el vestir o tener el pelo largo o tener aros, las uñas pintadas va a afectar la inteligencia de los niños (Comentario 252, Medio 2).

La discusión frente a esta representación cognitiva e instrumental se relaciona con una visión del sistema educativo chileno como reproductor de la condición social. Los argumentos en contra de una educación centrada exclusivamente en el aprendizaje se vinculan a que la educación debe preparar para un campo social estático y predefinido, en el cual aspectos anexos al aprendizaje son necesarios.

La vida laboral no contempla la ley de inclusión.... es otro mundo... (Comentario 22, Medio 1).

Espero que cuando ese "educando" esté trabajando y quiera "imponer" ese estilo libre e inclusivo carente de toda norma de vestir, simplemente no podrá trabajar ... Bueno, después de todo... es hijo de la inclusión (Comentario 116, Medio 1).

Si bien podría esperarse un referente de transformación social respecto de la función de la educación en los sujetos que aprueban la política inclusiva, los comentarios que evidencian esta representación sostienen una visión de educación que prepara para la vida en sociedad.

Son jóvenes, tienen que aprovechar ahora, antes de que los pille la máquina (de los adultos) donde ya será casi imposible (Cita 259, Medio 2).

Somos jóvenes el llamado es a que nos comprendan. Ya llegara el momento en que tengamos que trabajar y comportarnos como adultos, pero por ahora a disfrutar de esta linda etapa (Cita 202, Medio 2).

La representación cognitiva de que la educación tiene un rol de reproducción social constituye una base para valorar la disciplina y el orden en las escuelas. Se avala así un rol regulador de la imagen y comportamiento del estudiante, lo cual tensiona con una representación sobre la identidad que no puede privarse por estas condicionantes.

Se perderán las reglas básicas que se aprenden en un colegio, la disciplina... Después ese mismo estudiante, cuando entre a trabajar, irá como quiere al laburo (Comentario 27, Medio 1).

Los jóvenes hacen lo que quieren por la misma razón, no acatan órdenes. Sin ley, no hay orden (Comentario 358, Medio 4).

Una idea que refuerza y complementa la representación de la educación como derecho es que la escuela debiera respetar la identidad de los estudiantes, lo que además se asocia a un clima más apropiado para el aprendizaje, reforzándose la orientación hacia el aprender. 
Pero que de lo mismo lo del pelo largo o corto, pantalones anchos o pitillos, ya que lo único que hacen es bajarle el autoestima al alumno y eso afecta mucho en el sistema de aprendizaje de uno, ya que el alumno va a estar preocupado de cómo se ve (Comentario 160, Medio 2).

Siempre me molestó esa rigidez absurda que lo único que lograba era que mis alumnos se amargaran y se incentivara en ellos la rebeldía. Aquí no estamos hablando de algo extremo de ir sucio, o ser desubicado, simplemente de algo piola que te permite realizarte como persona (Comentario 264, Medio 2).

Contrario a la idea de una educación respetuosa de las identidades, existe una representación que entiende la exaltación de la identidad como una amenaza. En este sentido, se concibe que el fomento de la identidad podría aumentar las diferencias y, por tanto, la discriminación. Esta idea refuerza la representación reproductora de la sociedad en tanto la disciplina actuaría como mecanismo de control para evitar las diferencias arbitrarias, evadiéndose otras estrategias para hacer frente a este fenómeno.

Lo que realmente se necesita es disciplina y orden, hay unos cortes de pelos ridículos, bueno si el colegio no los echa los mismos compañeros lo harán, y los casos de bullyng escolar van a aumentar (Comentario 367, Medio 4).

Con esta ley es muy posible que se discriminen más niños, ya sea por las marcas, por el corte de pelo etcétera, mientras que si van con uniforme completo se van a ver iguales y no habría discriminación alguna (Comentario 159, Medio 2).

De esta forma, se releva el contrato social que se establece entre familia y escuela, el cual a través de sus proyectos educativos y reglamentos sustentan la autoridad para garantizar normas de comportamiento y favorecer el aprendizaje.

Por algo existe un reglamento interno, para el orden y la disciplina, y el respeto entre los alumnos y los funcionarios del establecimiento, lo único que harán es que los colegios dejen de lado los reglamentos internos y de convivencia, ya que no tendrán sentido, puesto que estarán anuladas por leyes del estado (Comentario 101, Medio 2).

Yo digo que esa ley le sigue quitando más autoridad a la escuela y los profesores se quedan sin herramienta para hacer cumplir la norma escolar del buen vivir en el establecimiento (Comentario 12, Medio 1).

\section{Discusión}

Las representaciones sobre la inclusión de las diferencias en educación se agrupan en dos modelos a analizar. El primero centrado en el derecho a la educación, y el segundo centrado en la necesidad de una educación que contemple el ámbito disciplinar en materia de conducta e identidad. 
Para el primer modelo (véase Tabla 3), la educación como derecho al aprendizaje, releva el rol de los objetivos y contenidos, para lo cual los aspectos de identidad, socioeconómicos y culturales de los estudiantes no constituyen elementos a intervenir. La regulación de estos aspectos por parte de las instituciones educativas, en lugar de constituir un facilitador para el aprendizaje, constituye una barrera en términos del clima escolar. Lo anterior es coherente con los planteamientos de Slee (2011), respecto de la política inclusiva, rechazando la normalización de los grupos a los patrones establecidos.

La política inclusiva que surge desde el respeto a la identidad se configura como una resistencia hacia la identidad oculta que se materializa en el conformismo frente a las exigencias académicas. De acuerdo con Bourdieu y Passeron (1963: 78), esta resistencia se concreta en la búsqueda de una diferencia que, en sí misma, constituye la "afirmación de la unanimidad de las aspiraciones estudiantes a un mismo nivel de vida, a la independencia y a una nueva relación pedagógica”. Lo interesante del modelo de educación como derecho identificado es que releva una relación pedagógica, que si bien no apunta a transformar la sociedad, considera en mayor medida las necesidades de los estudiantes que las de la estructura social.

El segundo modelo (véase Tabla 4) está constituido por la representación de la disciplina como aspecto que favorece el aprendizaje. En este sentido, el control sobre los estudiantes los prepara para la vida social, valorándose la homogeneidad, el orden y la estructura al interior de las escuelas. La problemática del comportamiento estudiantil fue anteriormente descrita por Bourdieu y Passeron (1963) como una condena a la cual el estudiante está sometido para proyectar lo que es en tanto tal.

Los estudiantes, a través de la "reacción contra la disciplina del secundario, se afirman como sujetos de un libre albedrío cultural" (Bourdieu y Passeron, 1963: 61). Así, resulta en vano descubrir modelos de comportamiento, toda vez que éstos se encuentran bajo conformismos de vestimenta, cosméticos e ideológicos que hacen que los estudiantes se parezcan entre sí, conformándose en torno a una única identidad.

Por otra parte, la valoración del orden y la homogeneidad en educación responde a una valoración de la disciplina como "primera iniciación a la austeridad del deber” (Durkheim, 2002: 129). Durkheim declara que la disciplina no constituye un mero acto para que el niño o la niña trabaje, su fin último es estimular la educación moral. Los desacuerdos frente a los planteamientos de identidad estudiantil contenidos en la Ley de Inclusión Escolar chilena son coherentes con este modelo, en tanto se establece la falta de disciplina como riesgo moral que conlleva a la inmoralidad, y ésta, a su vez, a la violencia. Así, 
la preocupación de la población es que al reducir las normas disciplinares y al perder poder los estamentos internos de la educación, se arriesgue el orden social.

Si se considera el concepto de habitus de Bourdieu (1980), el traspaso de la educación a manos de la Armada y al Ministerio del Interior chileno, en el año 1973, habría contribuido a instalar un currículum ideologizado con principios nacionalistas (Moreno-Doña y Gamboa, 2014). En este sentido, la sociedad chilena ha estructurado, y arrastrado a lo largo de la historia, una representación de educación vinculada con la educación militar (uniforme, exaltación de los valores patrios, jerarquías, reglamentos y servicio al país).

Aun cuando el retorno a la democracia a principios de la década de 1990 modificó el campo social, las estructuras educativas rígidas no han contado con herramientas desde las expectativas sociales y la formación inicial docente para ajustarse a las nuevas condiciones.

Se comprende así una identidad homogénea para contribuir a la disciplina y probablemente a una unificación nacional, toda vez que la educación chilena parece ser visualizada como productora de orden y uniformidad, favoreciendo la apropiada inserción de estudiantes al mundo laboral. Esta representación ha sido cuestionada previamente por Montero (2006), en tanto la educación pierde su sentido de derecho social universal al convertirse en una mercancía para el acceso y la competencia en el mercado.

En este punto se confrontan ambas representaciones respecto de la política de inclusión educativa, una en la cual la educación tiene un enfoque de derecho, y otra en la que la educación tiene fines de inserción laboral y social. El problema surge cuando, al no considerar la educación como derecho, se dota de cierto estatus a un grupo de personas en desmedro de otros, privándoles de participación (Insulza, 2014). La representación centrada en la inserción laboral denota en sí misma valores de competencia que necesariamente implican que el más competente es más valioso que el otro menos competente (Sennett, 2006), lo cual conlleva al desmedro de ese otro.

Durkheim (2002) también es crítico con este rasgo competitivo y propio de las sociedades capitalistas, toda vez que el individuo no puede desprenderse de la colectividad con la finalidad de alcanzar sus propias metas, pues, de hacerlo, se expone al abandono y al suicidio. Quienes plantean una desaprobación a la política inclusiva explicitan su interés por una educación preparadora, mientras que quienes la aprueban reconocen la escolaridad como un momento único de libertad, donde lo importante es aprender, para de todas formas insertarse luego en el mundo competitivo. 
De esta forma, los cambios económicos que ha sufrido la sociedad han promovido un ideal de sujeto caracterizado por la eficiencia, improvisación para la vida a corto plazo, la renovación de sus propias capacidades y la renuncia al pasado (Senett, 2006). La educación moral de la cual hace eco Durkheim (2002) queda incapacitada frente a un ideal de sujeto que, además de negar al otro, lo combate como su enemigo.

El rol de la educación en este contexto es clave, pues se ha establecido que el currículum educativo formula la naturaleza del hombre y de lo que se espera que éste sea (Roth, 1970). Por tal motivo, las estructuras bajo las cuales la sociedad chilena ha sido educada han promovido un modelo de hombre y mujer orientado al orden y la homogeneidad, lo cual sustenta una visión amenazadora de la identidad individual.

La discusión entre ambos modelos encuentra sustento en los planteamientos de Ainscow et al. (2013) y Belavi y Murillo (2016), quienes defienden la educación crítica que contribuye a hacer participar a docentes y estudiantes. La finalidad es desarrollar una cultura organizacional que comprenda de forma positiva la diversidad interrogando fenómenos y convirtiéndose en agentes de cambio. Así entendido, la Ley de Inclusión Escolar chilena encuentra como oportunidad de discusión la reconceptualización de la escuela, orientada hacia una formación crítica de estudiantes, que posteriormente se incorporen para transformar la sociedad más que persistir en la idea de una escuela pasivamente preparadora.

Esta polarización da cuenta de la necesidad de que la política pública no sólo se implemente, sino que además tenga instancias de participación y discusión en torno a las representaciones que le subyacen (Jobert, 2004). Lo anterior, entendiendo que a lo largo de la historia se han configurado sistemas de comprensión y actuación en la realidad. Asimismo, es interesante develar que la representación común que sustenta la educación chilena es la de una educación que prepara a los estudiantes para un mundo laboral ya estructurado e inmutable.

El artículo planteado pone de manifiesto la relevancia de estudiar las representaciones en torno a los cambios que la política educativa requiere. Esto permite profundizar en los sistemas cognitivos, instrumentales y valorativos que subyacen a la resistencia a los cambios de tal forma de actuar sobre ellos, a fin de promover transformaciones educativas sentidas y consensuadas con la población que las recibe. La necesidad de que la política educativa sea discutida y comprendida desde sus fines es un aspecto fundamental para modificar la visión que se tiene del rol de las instituciones educativas y, por tanto, de la estructura estática de la sociedad chilena. 
Jorge Eduardo Alfaro-Urrutia. Representaciones que subyacen a la politica educacional inclusiva en Chile

\section{Referencias}

Aguilar, Luis (2000), La implementación de las politicas, México: Porrúa.

Ainscow, Mel (2005), "Developing inclusive education systems: what are the leves for change?”, en Journal of Educational Change. Disponible en: http://link.springer.com/ article/10.1007/s10833-005-1298-4 [15 de agosto de 2016].

Ainscow, Mel et al. (2013), "De la exclusión a la inclusión. Una revisión literaria internacional en camino para responder a los estudiantes con necesidades educativas especiales en las escuelas", en En-clave Pedagógica, España: UH. Disponible en: http://hdl.handle. net/10272/8169 [02 de julio de 2016].

Araujo, Kathya y Martuccelli, Danilo (2015), "La escuela y la cuestión del mérito: reflexiones desde la experiencia chilena”, en Educacao e Pesquisa, Brasil: USP. Disponible en: http:// www.scielo.br/pdf/ep/v41 nspe/1517-9702-ep-41-spe-1503.pdf [05 de marzo de 2016].

Belavi, Guillermina y Murillo, Francisco (2016), "Educación, democracia y justicia social”, en Revista Internacional de Justicia Social, España: UAM. Disponible en: https:// revistas.uam.es/riejs/article/view/4371/4805 [17 de agosto de 2016].

Bellei, Cristian (2013), "El estudio de la segregación socioeconómica y académica de la educación chilena”, en Estudios Pedagógicos, año 39, num. 1, Chile: UACH. Disponible en: http://www.scielo.cl/scielo.php?script=sci_arttext\&pid =S0718-07052013000100019 [05 de marzo de 2016].

Bellei, Cristian (2015), El gran experimento. Mercado y privatización de la educación Chilena, Chile: LOM.

Blanco, Rosa (2014), "Inclusión educativa en américa latina: caminos recorridos y por recorrer”, en Marchesi, Álvaro et al. [coords.], Avances y desafíos de la educación inclusiva en Iberoamérica, España: OEI.

Booth, Tony (1995), "A perspective on Inclusion from England", en Cambridge Journal of Education, año 26, núm. 1, Reino Unido: Cambridge. Disponible en http://www. tandfonline.com/doi/abs/10.1080/0305764960260107? journalCode=ccje20 [05 de marzo de 2016].

Bourdieu, Pierre y Passeron, Jean-Claude (1963), Los herederos, los estudiantes y la cultura, Argentina: Siglo XXI.

Bourdieu, Pierre (1997), Capital cultural, escuela y espacio social, Argentina: Siglo XXI.

Bourdieu, Pierre (1980), El sentido práctico, Argentina: Siglo XXI.

Callén Blanca, et al. (2007), "Apuntes epistemo-políticos desde una etnografía tecnoactivista”, en Forum: Qualitative Social Research, año 8, núm. 3, Alemania: FUB. Disponible en http://www.qualitative-research.net/index.php/fqs/article/view/276/607 [04 de julio de 2017]

Capriati, Alejandro (2015), "Desigualdades y vulnerabilidad en la condición juvenil: el desafío de la inclusión social”, en Convergencia Revista de Ciencias Sociales, año 69, núm. 4, México: UAEM. Disponible en: http://convergencia.uaemex.mx/article/ view/3638 [16 de julio de 2016].

Castillo, Patricia et al. (2011), "Gestión y efectividad en educación: Evidencias comparativas entre establecimientos municipales y particulares subvencionados”, en Estudios pedagógicos, Chile: UACH, año 37, núm. 1. Disponible en http://www.scielo.cl/scielo. php?script=sci_arttext\&pid=S0718-07052011000100010 [05 de marzo de 2016]. 
Cavieres, Eduardo (2014), "La calidad de la educación como parte del problema. Educación escolar y desigualdad en Chile", en Revista Brasileira de Educacao, año 19, núm. 59, Brasil: ANPEd. Disponible en: http://www.scielo.br/scielo.php?script=sci_arttext\& pid=S1413-24782014000900011 [16 de julio de 2016].

Corvalán, Javier (2013), "Las dimensión es relacionales y simbólicas de los sistemas educativos: hipótesis para el caso chileno”, en Atenea, año 11, núm. 2, Chile: UdeC. Disponible en: http://www.scielo.cl/scielo.php?script=sci_arttext\&pid $=$ S0718-04622013000200009 [16 de julio de 2016].

Cox, Cristian (2012), "Política y políticas educacionales en Chile 1990-2010", en Revista Uruguaya de Ciencia Política, año 21, núm. 1, Uruguay: UR. Disponible en: http:// www.redalyc.org/pdf/2973/297325499002.pdf [15 de julio de 2016].

Crotty, Michael (1998), The Foundations of Social Research. Meaning an Perspective in the Research Process, Reino Unido: Sage.

Donoso-Díaz, Sebastián et al. (2012), "Visiones dominantes de la política educacional en la transición chilena hacia la democracia (1990-2010)", en Journal de Politicas Educacionais, año 12, núm. 1, Brasil. Disponible en: http://www.jpe.ufpr.br/n12_7. pdf [05 de marzo de 2016].

Donoso-Díaz, Sebastián y Arias, Óscar (2012), "Distribución desigual de las oportunidades educativas en el territorio y migración de la matrícula escolar: el caso de la región de los Lagos (Chile)", en Estudios Pedagógicos, año 38, núm. 2, Chile: UACH. Disponible en: http://www.scielo.cl/scielo.php?script=sci_arttext\&pid $=$ S0718-07052012000200003 [06 de julio de 2016].

Dubet, Fracoise (2014), Repensar la justicia social. Contra el mito de la igualdad de oportunidades, Argentina: Siglo XXI.

Durkheim, Emilio (2002), La educación moral, España: Morata.

Elliot, J. (2005), El cambio educativo desde la investigación-acción, España: Morata.

Giroux, Henry (1983), Teoría y resistencia en educación. Una pedagogía para la oposición, México: Siglo XXI.

Gurdián-Fernández, Alicia (2007), El paradigma cualitativo en la Investigación SocioEducativa, Costa Rica: Investigación y Desarrollo Educativo Regional.

Henríquez, Guillermo (2002), "El Uso de Herramientas de Internet en la Investigación Social”, en Cinta Moebio, año 13, núm. 1, Chile: UChile. Disponible en: http://www. facso.uchile.cl/publicaciones/moebio/13/henriquez.htm [16 de julio de 2016].

Hine, Christine (2004), Etnografía cultural. Nuevas tecnologias y sociedades, España: UOC. Infante, Marta (2010), "Desafíos de la formación docente: inclusión educativa”, en Estudios Pedagógicos, año 36, núm. 1, Chile: UACH. Disponible en: http://www.scielo.cl/ scielo.php?script=sci_arttext\&pid=S0718-07052010000100016 [02 de febrero de 2016].

Insulza, José (2014), “Inequality, Democracy and Social Inclusion”. Disponible en https:// www.oas.org/docs/desigualdad/LIBRO-DESIGUALDAD-INGLES.pdf $[26$ de febrero de 2016].

Jobert, Bruno (2004), Estado, sociedad, politicas públicas, Chile: Serie Universitaria.

Ley 20845 (2016), Diario Oficial de la República de Chile, Santiago, Chile (01 de marzo de 2016).

Margulis, M. (1999), “La discriminación en la discursividad social”, en Margulis, Mario et al. [comps.], La segregación negada. Cultura y discriminación social, Argentina: Biblos. 
Jorge Eduardo Alfaro-Urrutia. Representaciones que subyacen a la politica educacional inclusiva en Chile

Martínez, Juan (2013), “La viabilidad de una investigación cualitativa online: estudio de caso basado en la alfabetización mediática existente en trabajadores españoles expatriados", en Actas del Primer Congreso Internacional Comunicación y Sociedad. Disponible en: https://dialnet.unirioja.es/servlet/articulo?codigo=4249402 [17 de julio de 2016].

McMaster, Christopher (2015), "Where is___?: Culture and the process of change in the development of inclusive education", en International Journal of Whole Schooling, año 11, núm. 1. Disponible en: http://www.wholeschooling.net/Journal_of_Whole_ Schooling/articles/11-1\%20McMaster.pdf [05 de febrero de 2016].

Mehan, Hugh (2008), "A sociological perspective on opportunity to learn and assessment", en Moss, Pamela et al. [eds.], Assessment, Equity and Opportunity to Learn, Estados Unidos: Cambridge.

Mittler, Peter (1995), "Special needs education: an international perspective”, en British Journal of Special Education, año 22, núm. 3. Disponible en: http://onlinelibrary.wiley. $\mathrm{com} /$ doi/10.1111/j.1467-8578.1995.tb00915.x/abstract [15 de febrero de 2016].

Montero, Alberto (2006), "Educación, economía y mercado: crónica de una difícil relación”, en Educación XXI, año 9, núm. 1, España: UNED. Disponible en: http://www.redalyc. org/articulo.oa?id=70600901 [15 de marzo de 2016].

Moraga, Patricio (2015). "Discusión Social y parlamentaria de la Ley de Inclusión Escolar. Educación para la inclusión: No al lucro, no al copago, no a la selección”, Tesis para optar al grado de Magíster en Educación mención gestión educativa, Chile: Universidad Metropolitana de Ciencias de la Educación.

Moreno-Doña, Alberto y Gamboa, Rodrigo (2014), "Dictadura chilena y Sistema escolar: 'a otros dieron de verdad esa cosa llamada educación”, en Educar em Revista, vol. 51, núm. 1, Brasil: UFPR. Disponible en: http://www.scielo.br/pdf/er/n51/n51a05.pdf [15 de julio de 2016].

OECD (2015), Todos Juntos ¿Por qué reducir la deisgualdad nos beneficia?... en Chile. Disponible en: http://www.oecd.org/chile/OECD2015-In-It-Together-HighlightsChile.pdf [30 de enero de 2016].

OECD (2016), Fomentando un crecimiento inclusivo de la productividad en América Latina. Disponible en: http://www.oecd.org/economy/fomentando-un-crecimientoinclusivo-de-la-productividad-en-america-latina.pdf [05 de febrero de 2016].

Oliva, María-Angélica (2010), "Política educativa chilena 1965-2009.¿Quéoculta esa trama?”, en Revista Brasileira de Educacao, año 15, núm. 44, Brasil: ANPEd. Disponible en: http://www.scielo.br/scielo.php?script=sci_arttext\&pid=S1413-24782010000200008 [15 de julio de 2016].

ONU (1948), Declaración Universal de los Derechos Humanos. http://www.ohchr.org/EN/ UDHR/Documents/UDHR_Translations/spn.pdf [29 de enero de 2016].

Orellana, Dania y Sánchez, María-Cruz (2006), “Técnicas de recolección de datos en entornos virtuales más usadas en la investigación cualitativa”, en Revista de Investigación Educativa, año 24, núm. 1, España: RIE. Disponible en: http://revistas.um.es/rie/ article/view/97661 [12 de junio de 2016].

Ortiz, Iván (2014), "Adolescentes chilenos de estratos sociales superiores con baja competencia lectora”, en Estudios sobre Educación, año 26, núm. 1, España: UN. Disponible en: https://www.unav.edu/publicaciones/revistas/index.php/estudiossobre-educacion/article/view/1863 [15 de julio de 2016]. 
Peña, Mónica (2013), “Análisis crítico de discurso del Decreto 170 de Subvención Diferenciada para necesidades educativas especiales: El diagnóstico como herramienta de gestión”, en Psicoperspectivas, vol. 12, núm. 2, Chile: UMCE. Disponible en: http:// www.psicoperspectivas.cl/index.php/psicoperspectivas/article/view/252 [05 de febrero de 2016].

Quijano, Anibal (2014), Des/colonialidad y bien vivir. Un nuevo debate en Latinoamérica, Perú: Universitaria.

Roth, Eldon (1970), “Conceptions of the curriculum”, en Improving college and university teaching, año 18, núm. 2, Canadá. Disponible en: http://www.tandfonline.com/doi/ abs/10.1080/00193089.1970.10532938 [15 de marzo de 2016].

Sade-Beck, Liav (2004), "Internet ethnography: Online and offline", en International Journal of Qualitative Methods, año 3, núm. 2, Canadá: UAB. Disponible en: https:// sites.ualberta.ca/ iiqm/backissues/3_2/pdf/sadebeck.pdf [16 de julio de 2016].

Salend, Spencer (2011), Creating inclusive classrooms: Effective and reflective practices, Estados Unidos: Pearson.

Sacristán, Jimeno (2010), “QQué significa el currículum? (adelanto)”, en Sinéctica, vol. 34, México: ITESO. Disponible en: http://www.scielo.org.mx/scielo.php?script=sci_ arttext\&pid=S1665-109X2010000100009 [15 de febrero de 2016].

Salvat, Pablo (2009), "De la desigualdad y los desafíos para una sociedad justa: ¿cómo vivir juntos?”, en Cárcamo, Solange [ed], Justicia Social y Diversidad, Chile: Universidad Católica de Temuco.

Santos, Boaventura (2005), El milenio huérfano. Ensayos para una nueva cultura politica, Colombia: Trotta/Isla.

Santos, Boaventura (2010), Descolonizar el saber, reivindicar el poder, reinventar el poder, Chile: Trilce.

Schiro, Michael (2008), Curriculum Theory: Conflicting visions and enduring concerns, Estados Unidos: Sage.

Sennett, Richard (2006), La cultura del nuevo capitalismo, España: Anagrama.

Slee, Roger (2011), La escuela extraordinaria: Exclusión, escolarización y educación inclusiva, España: Morata.

Slee, Roger (2012), "How do we make inclusive education happen when exclusión is a political predisposition?”, en International Journal of Inclusive Education, año 17, núm. 8, Australia: UOSA. Disponible en: http://www.tandfonline.com/doi/abs/10.1080/ 13603116.2011.602534 [15 de marzo de 2016].

Tarabini-Castellani, Ainay y Sarró, Xavier (2011), “Globalización y política educativa: los mecanismos como método de estudio”, en Revista de Educación, núm. 355, España: MECD. Disponible en: https://dialnet.unirioja.es/servlet/articulo codigo=3638937 [05 de febrero de 2016].

Treviño, Ernesto et al. (2011), “'Segregar o incluir?: esa no debiera ser una pregunta en educación”, en Reflexiones Pedagógicas, año 45, núm. 1, Chile: Colegio de Profesores. Disponible en: http://www.revistadocencia.cl/new/wp-content/ pdf/20111216122143.pdf [05 de marzo de 2016].

Torres, Jurjo (2010), “La educación de la ciudadanía en una sociedad globalizada”, en Villalba, Felix y Villatoro, Javier [eds.], Aportaciones a la Educación Intercultural, España: Letras. 
Unesco (1990), "Declaración mundial sobre educación para todos y marco de acción para satisfacer las necesidades básicas de aprendizaje". Disponible en: http://www.unesco. org/education/pdf/JOMTIE_S.PDF [29 de enero de 2016].

Unesco (2005), "Políticas educativas de atención a la diversidad cultural Brasil, Chile, Colombia, México y Perú”. Disponible en: http://unesdoc.unesco.org/ images/0014/001470/147054s.pdf [29 de enero de 2016].

Unesco (2015), "EFA Global Monitoring Report 2015. Education for all”. Disponible en: http://unesdoc.unesco.org/images/0023/002322/232205e.pdf [15 de abril de 2015].

UNICEF (1989), "Convención sobre los derechos del niño". Disponible en http://www. unicef.org/ecuador/convencion(5).pdf [29 de enero de 2016].

Vásquez, María-Gabriela (2015), "La calidad de la educación. Reformas educativas y control social en América Latina”, en Latinoamérica, Revista de Estudios Latinoamericanos, año 60, núm. 1, México: UNAM. Disponible en: http://www.sciencedirect.com/science/ article/pii/S1665857415000046 [15 de marzo de 2016].

Villalobos, Cristóbal y Quaresma, María-Luisa (2015), "Sistema escolar chileno: características y consecuencias de un modelo orientado al mercado”, en Convergencia. Revista de Ciencias Sociales, año 69, núm. 4, México: UAEM. Disponible en http:// www.redalyc.org/articulo.oa?id=10540670003 [16 de marzo de 2016].

Welschinger, Nicolas (2012), "La etnografía virtual revisitada: Internet y las nuevas tecnologías digitales como objetos de estudio", en Revista Latinoamericana de Metodología de las Ciencias Sociales, año 2, núm. 2, Argentina: UNLP. Disponible en: http://sedici.unlp.edu.ar/handle/10915/26949 el [20 de agosto de 2015].

\section{Anexo}

\section{Tabla 1}

\section{Distribución de los comentarios y tipo de medio}

\begin{tabular}{clc}
\hline Medio & \multicolumn{1}{c}{ Tipo de medio } & Cantidad de comentarios \\
\hline 1 & Radio electrónica & 154 \\
\hline 2 & Periódico electrónico & 163 \\
\hline 3 & Periódico electrónico & 29 \\
\hline 4 & Noticiero electrónico & 28 \\
\hline 5 & Periódico electrónico & 04 \\
\hline & Total de casos & 378 \\
\hline
\end{tabular}

Fuente: Elaboración propia. 
Tabla 2

Sistematización de las representaciones respecto de la política de inclusión

\begin{tabular}{ll}
\hline \multicolumn{1}{c}{ Aprobación de la política } & \multicolumn{1}{c}{ Reprobación de la política } \\
\hline Centrado en el aprendizaje & Valoración de la disciplina y el orden \\
\hline Valoración del derecho a la educación & Preocupación por la discriminación \\
\hline Valoración del clima escolar & Reproducción social de la educación \\
\hline Fomento de la identidad & Proyecto educativo como contrato social \\
\hline
\end{tabular}

Fuente: Elaboración propia.

Tabla 3

\section{Modelo de Educación como derecho}

\begin{tabular}{lll}
\hline $\begin{array}{l}\text { Representaciones } \\
\text { Cognitivas }\end{array}$ & $\begin{array}{l}\text { Representaciones } \\
\text { Normativas }\end{array}$ & Representaciones Instrumentales \\
\hline $\begin{array}{l}\text { La educación debe } \\
\text { focalizarse en el apren- } \\
\text { dizaje }\end{array}$ & $\begin{array}{l}\text { Valoración de la iden- } \\
\text { tidad y la diferencia }\end{array}$ & $\begin{array}{l}\text { La escuela no debe preocuparse de } \\
\text { regular las diferencias para favore- } \\
\text { cer un buen clima escolar y así por } \\
\text { tanto el aprendizaje }\end{array}$ \\
\hline
\end{tabular}

Fuente: Elaboración propia.

Tabla 4

Modelo de educación como reproductora de la sociedad

\begin{tabular}{lll}
\hline $\begin{array}{l}\text { Representaciones } \\
\text { Cognitivas }\end{array}$ & $\begin{array}{l}\text { Representaciones } \\
\text { Normativas }\end{array}$ & Representaciones Instrumentales \\
\hline $\begin{array}{l}\text { La educación debe } \\
\text { preparar el mundo del }\end{array}$ & $\begin{array}{l}\text { Valoración de la dis- } \\
\text { ciplina y la homoge- } \\
\text { trabajo }\end{array}$ & $\begin{array}{l}\text { La familia establece un contrato } \\
\text { social con la escuela que debe res- } \\
\text { petarse para garantizar una buena } \\
\text { educación }\end{array}$ \\
\hline
\end{tabular}

Fuente: Elaboración propia. 
Jorge Eduardo Alfaro-Urrutia. Magíster en Educación mención evaluación educacional. Estudiante de Doctorado en Ciencias de la Educación, Universidad de la Frontera, Temuco, Chile. Líneas de investigación: evaluación educativa e inclusión escolar. Publicaciones recientes: Illesca, Ricardo y Eduardo, Alfaro Jorge, "Aptitud física y habilidades cognitivas", en Revista Andaluza de Medicina del Deporte (en prensa) (2016); Alfaro, Jorge Eduardo y Juan Santibáñez, "Diseño y propiedades psicométricas de un instrumento para evaluar habilidades de comprensión lectora en estudiantes de tercer año básico”, en Estudios Pedagógicos, año 41, núm. 2, Chile: UACH (2015); Conejeros, Patricia, Eduardo Alfaro y Patricia Peña, "Propuesta de validación de la batería psicomotora Vitor Da Fonseca", en Revista Oficial del Colegio de Kinesiólogos de Chile, vol. 29, núm. 3, Chile: Colegio de Kinesiólogos de Chile (2010).

Recepción: 7 de diciembre de 2016.

Aprobación: 23 de junio de 2017. 
